\title{
A NAA collaborative study in white rice performed in seven Asian countries
}

\author{
J. H. Moon • M. Ebihara • B. F. Ni • \\ B. Arporn $\cdot$ P. Setyo $\cdot$ R. M. Theresia $\cdot$ \\ B. S. Wee $\cdot$ N. A. Abd. Salim • P. C. B. Pabroa
}

Received: 29 May 2011 / Published online: 26 June 2011

(C) Akadémiai Kiadó, Budapest, Hungary 2011

Since 2000, collaborative studies for applying NAA have been performed through the Forum for Nuclear Cooperation in Asia (FNCA) sponsored by the Japanese Government. White rice is a main food for Asians and thus was selected as a common target sample for a collaborative study in 2008. Seven Asian countries including China, Indonesia, Japan, Korea, Malaysia, Philippines and Thailand, are greatly concerned about the composition of arsenic, heavy metals, and essential trace elements and took part in this study. Rice samples were purchased and prepared by following a protocol that had been proposed for this study. Samples were analyzed by their own NAA

J. H. Moon $(\bowtie)$

Korea Atomic Energy Research Institute, Daeduck-Daero 989-111, Duckjin-Dong, Yuseong, Daejeon, Korea

e-mail: jhmoon1@kaeri.re.kr

M. Ebihara

Department of Chemistry, Tokyo Metropolitan University, Hachioji, Tokyo 192-0397, Japan

B. F. Ni

China Institute of Atomic Energy, Beijing, People's Republic of China

B. Arporn

Thailand Institute of Nuclear Technology, Bangkok, Thailand

P. Setyo - R. M. Theresia

National Nuclear Energy Agency, Serpong, Indonesia

B. S. Wee $\cdot$ N. A. Abd. Salim

Malaysian Nuclear Agency, Bangi, 43000 Kajang,

Selangor, Malaysia

P. C. B. Pabroa

Philippine Nuclear Research Institute, Quezon City, Philippines systems. In each country, more than 10 elements were examined and the results were compared. These data will be very useful in the monitoring of the levels of food contamination and to evaluate the nutritional status for people living in Asia.

Keywords White rice - Inorganic elements - Neutron activation analysis - Asian countries - Dietary intake level

\section{Introduction}

The Forum for Nuclear Cooperation in Asia (FNCA) funded by the Japanese government was established to be recognized as an effective mechanism for enhancing socioeconomic development through active regional partnership in the peaceful and safe utilization of nuclear technology in 2000. Collaborative studies applying neutron activation analysis (NAA) have been carried out as one of FNCA projects for applications of nuclear technology for socioeconomic development $[1,2]$.

In the FNCA workshop held in Dalat, Vietnam in 2008, seven Asian countries, China, Indonesia, Japan, Korea, Malaysia, Philippines and Thailand, agreed to participate in a food sample analysis as a sub-theme of the NAA project. Rice was chosen as a common target sample due to it's importance in terms of a staple food for people living in the Asian region. That is, the major rice production countries in Asia are China, India, Indonesia, Bangladesh, Thailand, etc., and they account for more than 80 percent of the world's rice production and consumption. It implies that rice provides important information of mineral supplement as well as a large portion of calories for Asians. As scientists have focused their research on health impacts caused by mineral nutrient deficiency and hazardous 